\title{
Structural Degradations in the Bulk of Cathode Particles for Li-ion Batteries
}

\author{
Hanlei Zhang ${ }^{1,2}$, Fredrick Omenya ${ }^{2}$, M. Stanley Whittingham ${ }^{2}$, Chongmin Wang ${ }^{3}$, Guangwen Zhou ${ }^{1,2}$
}

1. Materials Science and Engineering Program \& Department of Mechanical Engineering, State University of New York, Binghamton, New York 13902, United States

2. NorthEast Center for Chemical Energy Storage, State University of New York, Binghamton, New York 13902, United States

3. Environmental Molecular Sciences Laboratory, Pacific Northwest National Laboratory, Richland, Washington 99352, United States

Layered oxides with an R-3m structure are promising cathode materials for lithium ion batteries (LIBs) for high rate capability and high energy density. However, the layered oxides suffer from a layered $\rightarrow$ rock-salt phase transformation that is believed to result in a "core-shell" structure of the primary particles, in which the core region maintains as the layered phase while the surface region transforms to the electrochemically inactive rock-salt phase. Using transmission electron microscopy, we demonstrate the formation of the rock-salt phase in the bulk of cycled primary particles with a formula of $\mathrm{LiNi}_{0.80} \mathrm{Co}_{0.15} \mathrm{Al}_{0.05} \mathrm{O}_{2}$ (NCA), in the form of rock-salt domains and rock-salt platelets, serving as sources for degraded electrochemical performance and intra-granular cracking of cathode particles, respectively.

Rock-salt domains of 50-70 nm are observed in the bulk of 30-cycle NCA particles, in which the surface and subsurface regions remain as the layered structure while the rock-salt phase forms as domains in the bulk with a thin layer of the spinel phase between the rock-salt core and the skin of the layered phase. These rock-salt domains are termed as an "anti-core-shell" structure in contrast to the well-established "core-shell" structure. Formation of this anti-core-shell structure is attributed to the oxygen loss at the surface that drives the migration of oxygen from the bulk to the surface, thereby resulting in localized areas of significantly reduced oxygen levels in the bulk of the particle, which subsequently undergoes the phase transformation to the rock-salt domains. The formation of the anti-core-shell rock-salt domains can lead to the reduced capacity, discharge voltage and ionic conductivity in cycled cathode.

Propagation and fracturing of platelet-like rock-salt phase along the (003) plane of the layered oxide are also observed in the bulk of cycled NCA particles, which is attributed to be the leading cause for intragranular cracking of primary particles. Rock-salt platelets at different developing stages (unfractured, partially fractured and fully fractured) are observed, confirming its leading role in the intra-granular cracking of cathode particles. The fracturing of the rock-salt platelet is induced by the stress discontinuity between the parent layered oxide and the rock-salt phase, which is a cyclic stress shock induced by the nonuniform lattice parameter changes between the layered and rock-salt phases during the electrochemical cycling. The high nickel content of NCA is considered to be the key factor for the formation of the rock-salt platelet and thus the (003) cracking. The (003)-type cracking can be a major factor for the structural degradation and associated capacity fade of the layered cathode.

\section{References:}

[1] Zhang, H.; Karki, K.; Huang, Y.; Whittingham, M. S.; Stach, E. A.; Zhou, G. The Journal of Physical Chemistry C 2017, 121, (3), 1421-1430. 
[2] Zhang, H.; Omenya, F.; Whittingham, M. S.; Wang, C.; Zhou, G. ACS Energy Letters 2017, 2, 2598-2606.

[3] Zhang, H.; Omenya, F.; Yan, P.; Luo, L.; Whittingham, M. S.; Wang, C.; Zhou, G. ACS Energy Letters 2017, 2, 2607-2615.

[4] This work was supported as part of the NorthEast Center for Chemical Energy Storage (NECCES), an Energy Frontier Research Center funded by the U.S. Department of Energy, Office of Science, Basic Energy Sciences under Award \# DE-SC0012583.
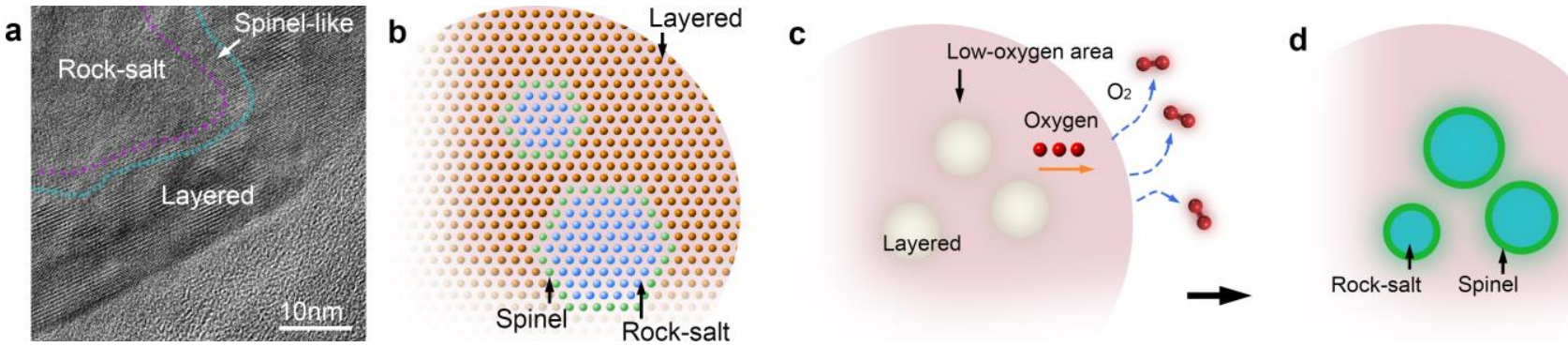

Figure 1. (a, b) HRTEM image and schematic showing the configuration of the anti-core-shell structure of the rock-salt domain that formed in the bulk of the primary particle. (c, d) Schematics showing the formation process of the anti-core-shell structure driven by the outward diffusion of oxygen from the bulk to the surface.
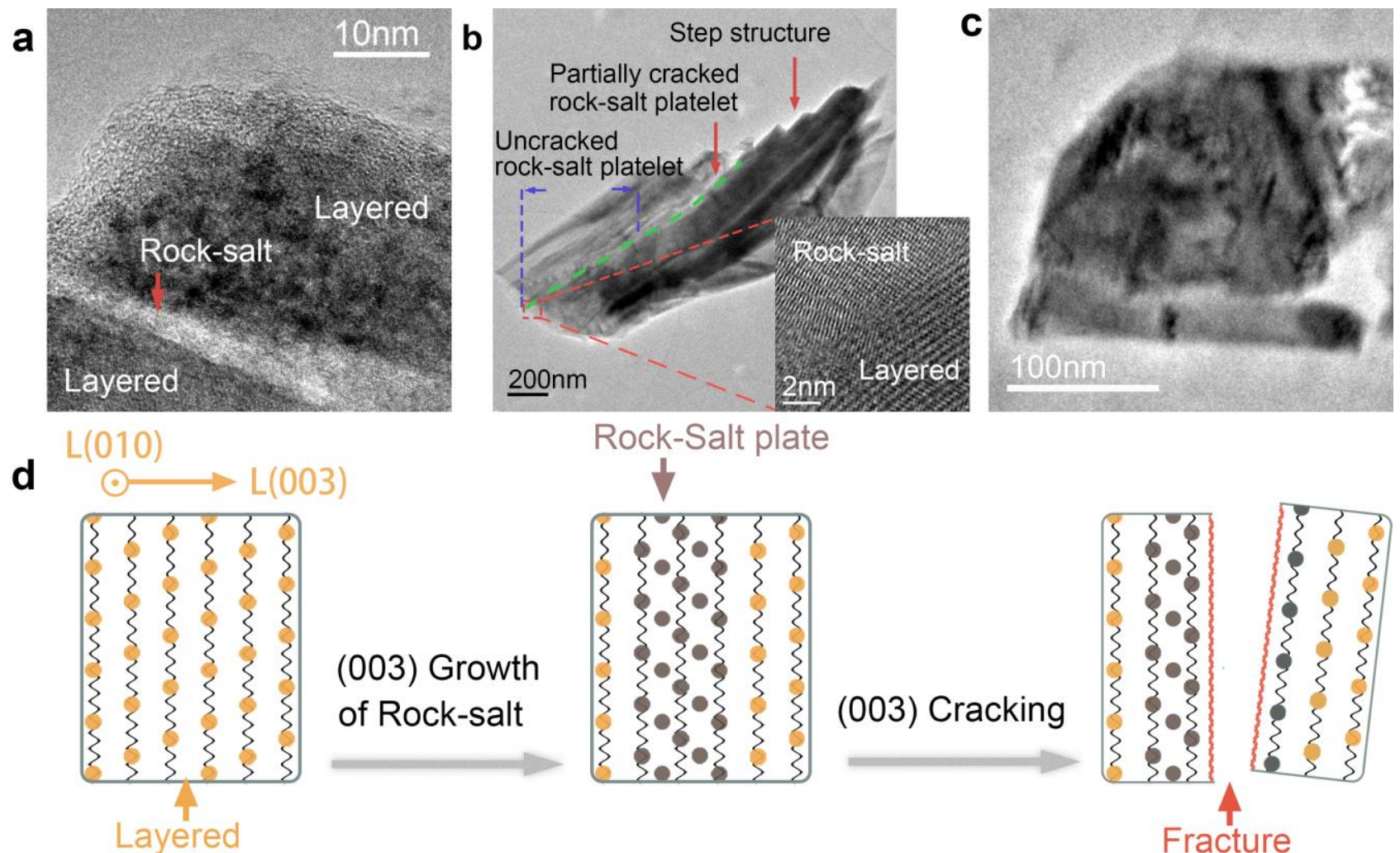

Figure 2. (a, b, c) HRTEM images showing unfractured, partially fractured and fully fractured rock-salt platelets, resulting in the development of (003)-type intra-granular cracking. (d) Schematic showing the formation and fracturing of (003)-type the rock-salt platelet. 